\title{
Another potential step to improve prosthetic heart valve endocarditis imaging with ${ }^{18} \mathrm{~F}-\mathrm{FDG}$ $\mathrm{PET} / \mathrm{CT}$
}

\author{
Thomas H. Schindler, $M^{a}$ \\ a Division of Nuclear Medicine, Cardiovascular Medicine, Department of Radiology and \\ Radiological Science, Johns Hopkins University School of Medicine, Baltimore, MD
}

Received May 22, 2017; accepted May 22, 2017

doi: 10.1007/s12350-017-0935-7

\section{See related article, pp. 1960-1967}

Scholtens et al. ${ }^{1}$ should be commended for their work to refine the imaging acquisition time for ${ }^{18}$ F-FDG PET/CT imaging for optimal diagnostic yield in the identification of prosthetic heart valve endocarditis (PVE). In thirteen patients with suspicion of PVE, standard ( $\approx 60$ minutes post i.v. injection) versus late ( $\approx 150$ minutes post i.v. injection) image acquisition of prosthetic heart valve FDG uptake with PET/CT proved to be less prone for false-positive results. As it was observed, visual analysis of FDG uptake with standard imaging resulted only in one false-negative and one false-positive finding as opposed to five false positive and zero false negative with the late imaging protocol. These observations, although low in numbers, add important information for establishing an optimal ${ }^{18} \mathrm{~F}$ FDG PET/CT imaging protocol for the detection of PVE. In general, for the identification and characterization of infection or inflammation, the standard image acquisition is applied based on the notion that there is a fast influx of glucose and, thus, FDG as glucose analog, into inflammatory cells followed by efflux dependent on the activity of glucose-6-phosphatase. Conversely, the late image acquisition assumes a persistent glucose influx in inflammatory activated cells and further clearance of glucose or FDG from the blood pool

Reprint requests: Thomas Hellmut Schindler, MD, Division of Nuclear Medicine, Cardiovascular Medicine, Department of Radiology and Radiological Science SOM, Johns Hopkins University, JHOC 3225, 601 N. Caroline Street, Baltimore, MD, 21287; tschind3@jhmi.edu, thomas.h.schindler@gmail.com

J Nucl Cardiol 2018;25:1968-70.

$1071-3581 / \$ 34.00$

Copyright (C) 2017 American Society of Nuclear Cardiology. yielding a higher contrast between activity in infected regions and background. ${ }^{2}$ Previous investigations ${ }^{2,3}$ have indeed suggested late imaging to add diagnostic value in the identification of infected cardiovascular implants. As the authors point out, the case description reported by Caldarella ${ }^{2}$ are in agreement with the one false-positive finding in the current investigation on late imaging. Yet, current observations ${ }^{1}$ stress that applying late image to negative standard image acquisition is likely to result in false-positive findings when the final diagnosis was based on surgical findings $(n=6)$ and unremarkable follow-up $(n=8)$. The latter reference of surgical findings and unremarkable follow-up may be seen as a strong reference for the current observations, ${ }^{1}$ which indeed argues late image acquisition as potential source of false-positive results in the detection of PVE with FDG PET/CT. It is also quite possible, however, that the optimal time point for ${ }^{18} \mathrm{~F}-\mathrm{FDG}$ PET/CT imaging in PVE may be found between 60 and 150 minutes depending on confounding factors such as diabetes mellitus, early or advanced stage of inflammation and/or period after interventional or surgical implantation of the prosthetic valve that warrants further clinical investigations. It is also important to keep in mind that the detection of e.g. cardiac implantable electronic device lead infection may reflect a different inflammatory disease entity for which late image acquisition in fact appear to be more suitable. ${ }^{3-5}$ As regards the quantification of the FDG uptake, maximal standard unit value (SUV max) and target-to-background ratio (TBR) between standard and late image acquisition unraveled a great variability in the individual measurements resulting in a wide overlap between both groups with PVE and non-PVE. ${ }^{1}$ Such finding emphasizes that even uninfected PHVs may have a certain amount of sterile inflammation, reflecting post-surgical tissue response and/or a mild foreign body reaction depending on the post-interventional period. Of interest, 
Saby et al. ${ }^{6}$ studied 72 consecutive patients with suspicion of having PVE with ${ }^{18}$ F-FDG PET/CT. They reported sensitivity, specificity, positive predictive value, negative predictive value, and diagnostic accuracy to be $73,80,85,67$, and $76 \%$, respectively. Importantly, adding abnormal ${ }^{18} \mathrm{~F}$ - FDG PET/CT imaging around the prosthetic valve as a major criterion significantly increased the sensitivity of the modified Duke criteria at admission from $70 \%$ to $97 \%$ owing to a marked reduction of possible PVE cases from 40 to 23 . Notably, the observed increase in sensitivity was obtained without comprising the specificity. For the time being, the modified Duke criteria provide the clinical reference for the identifying infective endocarditis based on clinical, echocardiographic, microbiological, and pathological findings. These criteria enable a diagnostic probability classification as definite, possible, or rejected endocarditis. ${ }^{7}$ Further, in the study of Saby et al., ${ }^{6}$ quantification of prosthetic valve FDG uptake with SUV max and TBR was highly variable among the three categories of definite, possible, or rejected PVE. While the mean value of SUV max was significantly higher in definite $\operatorname{PVE}(\approx 4.3)$ as compared to possible and rejected PVE, respectively ( $\approx 3.8$ and $\approx 3.6$ ), a high overlap among individual measurements widely ruled out the identification of a definite threshold to differentiate between true PVE, sterile inflammation, and no PVE. When looking at the TBR in these patients, it was mildly and non-significantly higher in definite PVE ( $\approx 2.0$ ) as compared to possible and rejected PVE, respectively $(\approx 1.8$ and $\approx 1.7)$. Thus, in line with the current observations of Scholtens et al., ${ }^{1}$ quantification of the FDG uptake 60 min post i.v. injection is highly variable in the individual patient with suspicion of PVE and, thus, should only be interpreted in the clinical context, such as medical history of the patients, clinical presentation, microbiological results, and imaging findings. There are important confounding factors in the assessment of PVE with ${ }^{18} \mathrm{~F}$-FDG PET/CT imaging that need to be given particular attention. The study of Saby et al. ${ }^{6}$ for example may have underestimated the falsepositive rate as the testing was in patients with high pretest probability for PVE. Thus, the diagnostic value of ${ }^{18}$ F-FDG PET/CT in the identification of PVE still needs further investigations in the clinically more relevant population with intermediate probability for the presence of PVE. Of note, early postoperative inflammation around the sewing ring of the prosthetic valve may lead to false-positive findings. This may also be seen as confounding factor in current results reported by Scholtens ${ }^{1}$ as ${ }^{18}$ F-FDG PET/CT imaging was performed in the range of 21-4992 days after prosthetic valve implantation. Other confounding factors that need to be taken into account such as unsuccessful suppression of myocardial FDG uptake despite optimal dietary preparation and prolonged fasting period of $>12 \mathrm{~h}^{8}$ Such unsuccessful suppression of myocardial FDG uptake may manifest very focal in the basal segment directly adjacent to the prosthetic valve leading to a false-positive finding. ${ }^{9}$ In addition, mild-to-moderate amount of FDG uptake around a prosthetic heart valve is commonly a normal finding likely owing to mild foreign body reaction and/or strain on the aortic wall. Other pitfalls of false-positive findings of PVE may be related to application of surgical adhesive during prosthetic valve implantation, the presence of atrial fibrillation with high energy consumption due to uncoordinated contraction of atrial myocytes, and lipomatous hypertrophy of the intertribal septum. ${ }^{9}$ Conversely, falsenegative findings may be related to preexisting effective antibiotic therapy associated with mild residual (nonsignificant) FDG uptake as well as vegetations on the prosthetic valve. ${ }^{9,10}$ Thus, apart from standard echocardiography, CT angiography of the valve should always be considered to exclude the presence of solitary vegetations. $^{10}$

In general, ${ }^{18}$ F-FDG PET/CT affords a high sensitivity in the identification of PVE when echocardiographic results are inconclusive. ${ }^{6,11}$ Yet, given the pitfalls leading to false-positive findings as described before, ${ }^{9}$ the specificity of ${ }^{18}$ F-FDG PET/CT in PVE detection may be limited, in particular within the first 2 months after surgical implantation. Albeit early identification of PVE is of utmost importance as these patients have a high mortality when not treated with geared antibiotics in a timely fashion, ${ }^{12}$ the low specificity of ${ }^{18}$ F-FDG PET/CT in PVE detection may lead to unnecessary surgical re-interventions not without risk. ${ }^{13}$ In this respect, leukocyte scintigraphy enables a high specificity for the presence of acute infection given radiolabeled leukocyte accumulation in the effected tissue. ${ }^{14}$ Rouzet et al. ${ }^{11}$ investigated in a head-to-head comparison the diagnostic of yield of ${ }^{18} \mathrm{~F}$ - FDG PET and leukocyte scintigraphy in 39 patients with suspicion of PVE. For ${ }^{18}$ F- FDG PET, the sensitivity, specificity, PPV, NPV, and accuracy proved to be $93 \%, 71 \%, 68 \%$, $94 \%$, and $80 \%$, respectively, while $64 \%, 100 \%, 100 \%$, $81 \%$, and $86 \%$, respectively, for leukocyte scintigraphy. ${ }^{11}$ Given these observations, it appears reasonable to perform ${ }^{18}$ F- FDG PET in patients with suspected PVE when results of echocardiography are not fully conclusive. While a negative ${ }^{18} \mathrm{~F}$ - FDG PET/CT widely rules out the presence of PVE, an enhanced FDG uptake adjacent and surrounding the prosthetic valve with a prosthetic valve-to-background ratio $>4.4$. is highly suggestive of PVE. ${ }^{11}$ When the FDG uptake is mild-tomoderate and diffuses around the prosthetic valve, however, the interpretation of the images remains 
uncertain. In such cases, the high specificity and PPV of leukocyte scintigraphy renders it as an important imaging asset to differentiate between PVE and postimplantation inflammatory process. ${ }^{11,14}$ The results of Scholtens et al. in the current issue ${ }^{1}$ further contribute to optimize the standard ${ }^{18} \mathrm{~F}$ - FDG PET in the identification of PVE by avoiding false-positive results with late imaging of the FDG uptake. Such observations, however, need to be confirmed in larger numbers and, in particular, also in patients with intermediate probability of PVE undergoing ${ }^{18} \mathrm{~F}-$ FDG PET/CT. Further improvements in dietary preparations in conjunction with may be several time points in FDG PET/CT image acquisition is expected to lead to a further refinement and improved diagnostic accuracy of ${ }^{18} \mathrm{~F}$ - FDG PET/CT in the identification of PVE.

\section{Disclosures}

None.

\section{References}

1. Scholtens A, Swart L, Verberne H, Budde R, Lam M. Dual time point FDG PET/CT imaging in prosthetic heart valve endocarditis. J Nucl Cardiol 2017 (Epub ahead).

2. Caldarella C, Leccisotti L, Treglia G, Giordano A. Which is the optimal acquisition time for FDG PET/CT imaging in patients with infective endocarditis? J Nucl Cardiol 2013;20:307-9.

3. Leccisotti L, Perna F, Lago M, Leo M, Stefanelli A, Calcagni ML, et al. Cardiovascular implantable electronic device infection: delayed vs standard FDG PET-CT imaging. J Nucl Cardiol 2014;21:622-32.

4. Memmott MJ, James J, Armstrong IS, Tout D, Ahmed F. The performance of quantitation methods in the evaluation of cardiac implantable electronic device (CIED) infection: A technical review. J Nucl Cardiol 2016;23:1457-66.

5. Tlili G, Amraoui S, Mesguich C, Riviere A, Bordachar P, Hindie E, et al. High performances of (18)F-fluorodeoxyglucose PET-CT in cardiac implantable device infections: A study of 40 patients. $\mathbf{J}$ Nucl Cardiol 2015;22:787-98.

6. Saby L, Laas O, Habib G, Cammilleri S, Mancini J, Tessonier L, et al. Positron emission tomography/computed tomography for diagnosis of prosthetic valve endocarditis: increased valvular $18 \mathrm{~F}-$ fluorodeoxyglucose uptake as a novel major criterion. J Nucl Cardiol 2013;61:2374-82.

7. Li JS, Sexton DJ, Mick N, et al. Proposed modifications to the Duke criteria for the diagnosis of infective endocarditis. Clin Infect Dis 2000;30:633-8.

8. Osborne MT, Hulten EA, Murthy VL, Skali H, Taqueti VR, Dorbala D, et al. Patient preparation for cardiac fluorine-18 fluorodeoxyglucose positron emission tomography imaging of inflammation. J Nucl Cardiol 2017;24:86-99.

9. Scholtens AM, Swart LE, Verberne HJ, Tanis W, Lam MG, Budde RP. Confounders in FDG-PET/CT imaging of suspected prosthetic valve endocarditis. JACC Cardiovasc Imaging 2016;9:1462-5.

10. Tanis W, Scholtens A, Habets J, et al. CT angiography and (1)(8)F-FDG-PET fusion imaging for prosthetic heart valve endocarditis. JACC Cardiovasc Imaging 2013;6:1008-13.

11. Rouzet F, Chequer R, Benali K, Lepage L, Ghodbane W, Duval X, et al. Respective performance of 18F-FDG PET and radiolabeled leukocyte scintigraphy for the diagnosis of prosthetic valve endocarditis. J Nucl Med 2014;55:1980-5.

12. Choussat R, Thomas D, Isnard R, Michel PL, Lung B, Hanania G, et al. Perivalvular abscesses associated with endocarditis; clinical features and prognostic factors of overall survival in a series of 233 cases. Perivalvular Abscesses French Multicentre Study. Eur Heart J 1999;20:232-41.

13. Rouzet F, Hyafil F, Le Guludec D. FDG PET/CT in cardiac electronic devices infection: Now is the time to target guidelines implementation. J Nucl Cardiol 2015;22:800-3.

14. Horgan SJ, Waller AH, Veeranna V, Kirshenbaum J, Dorbala S, Di Carli MF. Utility of multimodality imaging in suspected prosthetic valve endocarditis. J Nucl Cardiol 2016;23:316-20. 